\section{EUROPEAN MULTI-DISCIPLINARY TUMOUR BOARDS SUPPORT CROSS-BORDER NETWORKING AND INCREASE TREATMENT OPTIONS FOR PATIENTS WITH RARE TUMOURS}

${ }^{1} \mathrm{U}$ Joneborg ${ }^{*},{ }^{2} \mathrm{~A}$ Bergamini, ${ }^{1} \mathrm{E}$ Wallin, ${ }^{2} \mathrm{G}$ Mangili, ${ }^{3} \mathrm{O}$ Solheim, ${ }^{3} \mathrm{E}$ Rokkones, ${ }^{4} \mathrm{~A}$ Casado, ${ }^{4} \mathrm{G}$ Marquina, ${ }^{5} \mathrm{C}$ Lok, ${ }^{5} \mathrm{~N}$ Van Trommel, ${ }^{6} \mathrm{PA}$ Bolze, ${ }^{7} \mathrm{~J}$ Coulter, ${ }^{8} \mathrm{P}$ Pautier, ${ }^{9} \mathrm{~F}$ Goffin, ${ }^{10} \mathrm{~S}$ Han, ${ }^{9} \mathrm{~F}$ Kridelka, ${ }^{11}$ J Sehouli, ${ }^{5} \mathrm{~F}$ Amant, ${ }^{12}$ | Ray-Coquard, ${ }^{13} \mathrm{M}$ Seckl. ${ }^{1}$ Karolinska University Hospital, Department of Pelvic Cancer, Stockholm, Sweden; ${ }^{2} I R C C S$ San Raffaele Hospital, Department of Obstetrics and Gynecology, Milan, Italy; ${ }^{3}$ Norwegian Radium Hospital, Department of Gynecologic Oncology, Oslo, Norway; ${ }^{4}$ Hospital Universitario San Carlos, Department of Medical Oncology, Madrid, Spain; ${ }^{5}$ Antoni van Leeuwenhoek/ Netherlands Cancer Institute, Center for Gynecologic Oncology Amsterdam, Amsterdam, Netherlands; ${ }^{6}$ Hôpital Lyon Sud, Hospices Civils de Lyon, Service de Chirurgie Gynécologique et Oncologique, Obstétrique, Lyon, France; ' Cork University Hospital, National GTD Centre, Cork, Ireland; ${ }^{8}$ Institut Gustave-Roussy, Villejuif, France; ${ }^{9}$ University Hospital Liège, Department of Obstetrics and Gynecology, Liège, Belgium; ${ }^{10}$ University Hosppital Leuven, Department of Obstetrics and Gynecology, Leuven, Belgium; ${ }^{11}$ Charité Medical University of Berlin, Department of Gynecology with Center for Oncological Surgery, Berlin, Germany; ${ }^{12}$ Centre Leon Berard Lyon and GINECO, Lyon, France; ${ }^{13}$ Charing Cross Hospital Campus of Imperial College London, Gestational Trophoblastic Disease and Malignant Ovarian Germ Cell Tumour Centres, London, UK

\subsection{6/ijgc-2021-ESGO.273}

Introduction/Background* EURACAN is the European reference network for rare adult solid tumours coordinated by the French Comprehensive Cancer Centre Léon Bérard in Lyon, France. It consists of 10 domains, of which rare gynaecological tumours make up domain 2 (G2). Within the G2 domain, virtual multidisciplinary tumour boards (MDTs) were created to get support in clinical management of rare tumours. The aim of this report is to evaluate the outcomes in terms of participation, adherence to recommendations and access to novel patient treatment strategies.

Methodology EURACAN G2 cross-border MDTs were initiated in November 2017 and organized by Karolinska University Hospital, Stockholm, Sweden. The MDTs were held monthly at pre-set dates and G2 health care providers (HCP) were invited to present patient cases. A standardized form was used for patient data collection and summaries of cases to be

\begin{tabular}{ll} 
Abstract 602 Table 1 & Background data \\
\hline EURACAN MDT 2017-2020 & \\
$\mathrm{n}=91$ patients & \\
\hline Age median (IQR) & $37(27-52)$ \\
\hline Diagnosis $n$ (\%) & $19(21)$ \\
- GTD & $19(21)$ \\
- MOGCT & $13(14)$ \\
- SCST & $25(27)$ \\
- Rare ovarian & $9(10)$ \\
- Rare uterine & $2(2)$ \\
- Rare cervical & $4(4)$ \\
- Other & \\
Previous lines of treatment median (IQR) & $1(0-2)$ \\
Indication for discussion* $n(\%)$ & $59(54)$ \\
- Primary treatment & $7(6)$ \\
- Disease progression & $42(39)$ \\
- Relapse & $1(1)$ \\
- Other & \\
\hline
\end{tabular}

${ }^{*} \mathrm{n}=109$ individual case discussions

Abbreviations: GTD=gestational trophoblastic disease; MOGCT=malignant ovarian germ cell tumour; SCST=sex cord stromal tumour

\begin{tabular}{ll} 
Abstract 602 Table 2 Follow-up data & \\
\hline EURACAN MDT $2017-2020$ & \\
$\mathrm{n}=64$ patients & \\
\hline Adherence to treatment recommmendations* $n(\%)$ & $79(99)$ \\
- Yes & $1(1)$ \\
- No & \\
Treatment not otherwise available $n(\%)$ & $11(17)$ \\
- Off-label treatment & $1(2)$ \\
- Clinical trial abroad & \\
One-year follow-up $n(\%)$ & $28(44)$ \\
- Alive without disease & $16(25)$ \\
- Alive with disease & $5(8)$ \\
- Dead of disease & $15(23)$ \\
- One year not reached &
\end{tabular}

discussed was distributed to all HCPs before each MDT. A video- and telephone conference system was used for the first two years, and thereafter Webex meetings. After each MDT, a meeting summary with treatment recommendations was sent to all HCPs and the project manager at the coordinating centre. MDT format and outcomes were discussed at G2 domain meetings. Data regarding clinical characteristics of discussed cases and treatment recommendations were registered in a prospective database. Follow up data were retrieved until May 15, 2021.

Result(s)* Between November 2017 and December 2020, 31 MDTs were held with participants from 10 countries and 20 HCPs. 91 individual patients were discussed between one and six times for a total of 109 case discussions. Background data is presented in table 1. Follow-up data was retrieved from 64 patients and 80 case discussions (table 2). Adherence to treatment recommendations was 99\%. As a result of MDT recommendations, 11 patients got access to off-label treatment and one patient was enrolled in a clinical trial in another European country. 14/91 patients were recommended surveillance only.

Conclusion* Cross-border MDTs enable networking and clinical collaboration between health care professionals in different countries. Surveillance strategies, off-label drug use and participation in clinical trials in other countries are possible benefits to patients with rare gynecological tumours.

\section{PELVIC EXENTERATION FOR LOCALLY ADVANCED AND RELAPSED GYNECOLOGIC CANCER: MORBIDITY AND LONG-TERM SURVIVAL}

N Teixeira*, C Soler, R Luna Guibourg, P Español, R Rovira Negre. Hospital de la Santa Creu i Sant Pau, Barcelona, Spain

\subsection{6/ijgc-2021-ESGO.274}

Introduction/Background* Pelvic exenteration (PE) can be described as the most radical surgery performed in patients with recurrence or persistence of gynecologic cancer. The aim of this study was to evaluate morbidity and survival after PE. Furthermore we analyzed the impact of type of PE and age on survival.

Methodology This is a retrospective observational study including patients with histological diagnosis of gynecologic malignant pelvic tumor (cervix, ovary, endometrium, vulva, vagina, 